\title{
Magnetoresistance and Magnetic Properties of the Double Perovskites
}

\author{
J.B. Philipp, P. Majewski, D. Reisinger, S. Geprägs, \\ M. Opel, A. Erb, L. AlfF And R. Gross* \\ Walther-Meißner-Institut, Bayerische Akademie der Wissenschaften \\ Walther-Meißner Str. 8, 85748 Garching, Germany
}

\begin{abstract}
The magnetic double perovskite materials of composition $\mathrm{A}_{2} \mathrm{BB}^{\prime} \mathrm{O}_{6}$ with $A$ an alkaline earth ion and $B$ and $B^{\prime}$ a magnetic and non-magnetic transition metal or lanthanide ion, respectively, have attracted considerable attention due to their interesting magnetic properties ranging from antiferromagnetism to geometrically frustrated spin systems and ferromagnetism. With respect to application in spin electronics, the ferromagnetic double perovskites with $\mathrm{BB}^{\prime}=\mathrm{CrW}$, CrRe, FeMo, or FeRe and $\mathrm{A}=\mathrm{Ca}$, Ba, Sr are highly interesting due to their in most cases high Curie temperatures well above room temperature and their half-metallic behavior. Here, we summarize the structural, magnetotransport, magnetic, and optical properties of the ferromagnetic double perovskites and discuss the underlying physics. In particular, we discuss the impact of steric effects resulting in a distorted perovskite structure, doping effects obtained by a partial replacing of the divalent alkaline earth ions on the A site by a trivalent lanthanide as well as $\mathrm{B} / \mathrm{B}^{\prime}$ cationic disorder on the Curie temperature $T_{\mathrm{C}}$, the saturation magnetization and the magnetotransport properties. Our results support the presence of a kinetic energy driven mechanism in the ferromagnetic double perovskites, where ferromagnetism is stabilized by a hybridization of states of the non-magnetic $\mathrm{B}^{\prime}$-site positioned in between the high spin B-sites.
\end{abstract}

PACS numbers: 75.50.-y, 75.50.Cc, 75.50.Ss

\section{Introduction}

Double perovskites of composition $\mathrm{A}_{2} \mathrm{BB}^{\prime} \mathrm{O}_{6}$ with $\mathrm{A}$ an alkaline earth such as $\mathrm{Sr}, \mathrm{Ba}$, or $\mathrm{Ca}$ and $\mathrm{B}, \mathrm{B}^{\prime}$ a magnetic and non-magnetic transition metal or

*corresponding author; e-mail: Rudolf.Gross@wmi.badw.de 
lanthanide ion, respectively, have been known for many decades [1-5]. However, only recently the study of ordered double perovskite materials has been strongly intensified both by their interesting magnetic properties ranging from antiferromagnetism to geometrically frustrated spin systems and ferromagnetism and, more importantly, the discovery of a large room temperature magnetoresistive effect at low magnetic fields in the system $\mathrm{Sr}_{2} \mathrm{FeMoO}_{6}$ [6]. With respect to applications, the fact that some magnetic double perovskites seem to be ferromagnetic metals with high Curie temperatures $T_{\mathrm{C}}$ of up to $635 \mathrm{~K} \mathrm{[7]} \mathrm{and} \mathrm{apparently} \mathrm{have} \mathrm{a} \mathrm{highly}$ spin polarized conduction band makes these materials interesting for spintronic devices such as magnetic tunnel junctions, low-field magnetoresistive sensors or spin injection devices [8]. Up to now large low-field magnetoresistive effects have been found not only in $\mathrm{Sr}_{2} \mathrm{FeMoO}_{6}[6,9,10]$, but also in many other double perovskites as for example $\mathrm{Sr}_{2} \mathrm{FeReO}_{6}$ [11], $\mathrm{Sr}_{2} \mathrm{CrReO}_{6}$ [7], $\mathrm{Sr}_{2} \mathrm{CrWO}_{6}$ [12-14], and $\left(\mathrm{Ba}_{0.8} \mathrm{Sr}_{0.2}\right)_{2-x} \mathrm{La}_{x} \mathrm{FeMoO}_{6}$ [15]. With respect to fundamental aspects, the main research effort is focused on the understanding of the magnetic interactions in the various types of magnetic double perovskites and their relation to structural, electronic, as well as magnetotransport properties.

The rich physics of the double perovskites $\mathrm{A}_{2} \mathrm{BB}^{\prime} \mathrm{O}_{6}$ is related to the fact that they are very flexible with respect to variations of the magnetic and/or non-magnetic $\mathrm{BB}^{\prime}$ ions as well as the $\mathrm{A}$-site cations. In this way they offer a wide range of possibilities to influence the structure and/or the magnetic interactions and, hence, to tailor the magnetic properties for specific applications or fundamental studies:

1. The magnetic ion on the B-site can be varied using either $3 d$ or $4 d$ transition metals (e.g. Fe, Cr, Mn, Ni, Co, Ru) with localized states or $4 f$ lanthanides (e.g. Gd, Dy, Ho, Er) with highly localized states.

2. The non-magnetic ion on the $\mathbf{B}^{\prime}$-site can be varied. Here, we have to distinguish between ions with delocalized electrons (e.g. $\mathrm{Mo}^{5+}, \mathrm{W}^{5+}, \mathrm{Re}^{5+}, \mathrm{Re}^{6+}$ ) and those with a noble gas electronic configuration (e.g. $\mathrm{Nb}^{5+}, \mathrm{W}^{6+}$ ) or a completely filled $3 d$ - or $4 d$-shell (e.g. $\mathrm{Sb}^{5+}, \mathrm{Te}^{6+}$ ).

3 . We can dope electrons into the system by partially replacing the divalent alkaline earth ions on the $\mathrm{A}$-site by a trivalent rare-earth ion such as $\mathrm{La}^{3+}$ [14-16].

4. The crystal structure can be changed by using alkaline earth ions on the A-site with different ionic radii. This results in considerable steric effects (see Fig. 1b), which in turn change the bond angles thereby modifying the magnetic interactions.

5. We can also use magnetic ions on both the $\mathrm{B}$ - and $\mathrm{B}^{\prime}$-site as e.g. in $\mathrm{LaAVRuO}_{6}$ and $\mathrm{LaAVMn}_{6}$ with $\mathrm{A}=\mathrm{Ca}, \mathrm{Sr}, \mathrm{Ba}$, or $\mathrm{La}_{2} \mathrm{CrFeO}_{6}[17,18]$. This class of double perovskites, which we do not discuss here, may be interesting for the realization of half-metallic antiferromagnets [19]. 


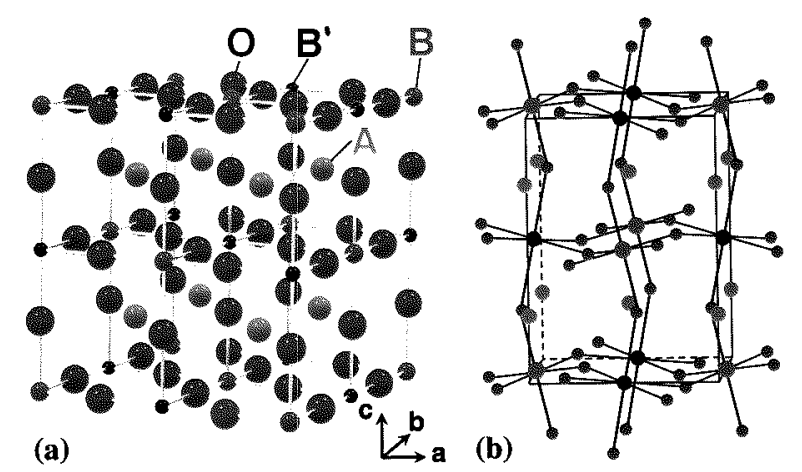

Fig. 1. Sketch of the crystal structure of a cubic double perovskite (a) with tolerance factor close to unity. In part (b) a distorted structure is shown. For a tolerance factor significantly deviating from unity the tilt and rotation of the $\mathrm{BO}_{6}$ octahedra result in a non-cubic crystal structure and $\mathrm{B}-\mathrm{O}-\mathrm{B}^{\prime}$ bonding angles deviating from $180^{\circ}$. The $\mathrm{B}$ and $\mathrm{B}^{\prime}$ ions are assumed to be perfectly ordered.

In this article, we discuss the magnetic interactions in the double perovskites with $\mathrm{B}$ - a magnetic and $\mathrm{B}^{\prime}$ - a non-magnetic ion, which are still discussed controversially. We summarize the present knowledge based on our own results and the data available in literature. The model considerations are compared to experimental data obtained for the system $\mathrm{A}_{2} \mathrm{CrWO}_{6}$ with $\mathrm{A}=\mathrm{Sr}, \mathrm{Ca}, \mathrm{Ba}$ and the electron doped system $\mathrm{Sr}_{2-x} \mathrm{La}_{x} \mathrm{CrWO}_{6}$.

\section{Magnetic interactions in the double perovskites}

The perovskites of composition $\mathrm{ABO}_{3}$ are ubiquitous in oxide chemistry. The perovskite structure in the simplest case consists of a $3 \mathrm{D}$ lattice of fully corner sharing $\mathrm{BO}_{6}$ octahedra forming a cavity, in which the larger atom $\mathrm{A}$ is 12 -fold coordinated to oxygen. Many variants are known. The double perovskites of composition $\mathrm{A}_{2} \mathrm{BB}^{\prime} \mathrm{O}_{6}$ have chemical supercells, in which two kinds of ions are found at the B-site in alternating octahedra (see Fig. 1a). A particularly interesting configuration, which we will discuss in the following, is the case of an ordered double perovskite, in which only the ion on the B-site carries a magnetic moment. In this case the ordered sublattices of the $\mathrm{B}$ and $\mathrm{B}^{\prime}$ ions form two interpenetrating fcc networks. In reality, of course, there is always a certain amount of B/ $\mathrm{B}^{\prime}$ cationic disorder (antisite defects), which may considerably affect the magnetic properties.

The magnetic interactions in the double perovskites with the B- and B'-site ions being magnetic and non-magnetic ions, respectively, are very interesting and complex. They strongly depend on the details of the crystal structure, the amount of $\mathrm{B} / \mathrm{B}^{\prime}$ antisites, and the magnetic and electronic properties of the $\mathrm{B}$ and $\mathrm{B}^{\prime}$ ions. In the following we discuss the various types of magnetic interactions dominating in different systems and classify the double perovskite materials into different 
categories accordingly. With respect to the magnetic ion on the B-site, we will distinguish the following two cases:

- the magnetic ion is a magnetic lanthanide ion with highly localized $4 f$ states such as $\mathrm{Gd}^{3+}, \mathrm{Dy}^{3+}, \mathrm{Ho}^{3+}$, or $\mathrm{Er}^{3+}$. There is a negligible hopping of the $4 f$ electrons preventing a hopping based magnetic interaction mechanism.

- the magnetic ion is a transition metal ion with less localized $3 d$ or $4 d$ states such as $\mathrm{Fe}^{2+}, \mathrm{Fe}^{3+}, \mathrm{Cr}^{3+}, \mathrm{Ni}^{2+}, \mathrm{Co}^{2+}, \mathrm{Mn}^{2+}, \mathrm{V}^{3+}$ or $\mathrm{Ru}^{4+}$. There is a sizable hopping resulting in a hopping based magnetic interaction mechanism, which depends on the electronic structure of the non-magnetic $\mathrm{B}^{\prime}$-site ion.

With respect to the non-magnetic ion on the $\mathrm{B}^{\prime}$-site, we will distinguish the following two cases:

- The non-magnetic $\mathrm{B}^{\prime}$-ion has a partially filled outer shell with one or two delocalized electrons (e.g. $\mathbf{M o}^{5+}, \mathrm{W}^{5+}, \mathrm{Re}^{5+}, \mathrm{Re}^{6+}$ ). Typical examples are $\mathrm{Sr}_{2} \mathrm{FeMoO}_{6}[6,9,10,20], \mathrm{Sr}_{2} \mathrm{CrReO}_{6}$ [7], $\mathrm{Sr}_{2} \mathrm{CrWO}_{6}$ [12-14] as well as $\mathrm{Sr}_{2} \mathrm{FeReO}_{6}[11,21]$ or $\mathrm{Ca}_{2} \mathrm{FeReO}_{6}[21-23]$.

- The non-magnetic $\mathrm{B}^{\prime}$-ion has a noble gas electronic configuration (e.g. $\mathrm{Nb}^{5+}$, $\mathrm{Ca}^{2+}, \mathrm{W}^{6+}$ ) or a completely filled $3 d$ - or $4 d$-shell (e.g. Sb ${ }^{5+}, \mathrm{Te}^{6+}$ ). Typical examples are $\mathrm{Sr}_{2} \mathrm{FeNbO}_{6}$ [24-26], $\mathrm{Sr}_{2} \mathrm{FeWO}_{6}$ [5, 27-29], $\mathrm{Ca}_{2} \mathrm{NiWO}_{6}$ and $\mathrm{Ca}_{2} \mathrm{CoWO}_{6}$ [30], $\mathrm{A}_{2} \mathrm{MnWO}_{6}$ with $\mathrm{A}=\mathrm{Ca}$, Sr, Ba [31-33], $\mathrm{Sr}_{2} \mathrm{ReCaO}_{6}$ [34], $\mathrm{Sr}_{2} \mathrm{NiTeO}_{6}$ [35], or $\mathrm{Sr}_{2} \mathrm{LnSbO}_{6}$ with $\mathrm{Ln}=\mathrm{Dy}, \mathrm{Ho}$, Gd [36].

\subsection{Double perovskites with $B=$ magnetic lanthanide ion}

In double perovskite systems $\mathrm{A}_{2} \mathrm{BB}^{\prime} \mathrm{O}_{6}$ with $\mathrm{B}$ - a magnetic lanthanide ion with highly localized $4 f$ states, any magnetic exchange based on the hopping of electrons such as antiferromagnetic superexchange of ferromagnetic double exchange cannot operate, since the probability for an electron to hop from a $4 f$ state at one site to another at a neighboring site is too small. This is independent of the type of ion on the $\mathrm{B}^{\prime}$-site. However, in this situation there may be a weak near-neighbor indirect exchange via the conduction electrons (Ruderman-KittelKasuya-Yoshida (RKKY) type interaction) or dipolar interactions. In this case the magnetic properties of the system may be very interesting due to frustration effects. For example, in the case of ordered sublattices of the B- and B'-ions the interpenetrating fcc networks fall into the class of geometrically frustrated spin systems, since an fcc lattice is composed of a network of edge-shared tetrahedra (see Fig. 2). If we assume that there is only a simple antiferromagnetic near-neighbor coupling among the magnetic B-ions, the magnetic sublattice consisting of edge-shared tetrahedra (see Fig. 2) is known to result in geometric magnetic frustration. Magnetic materials in which long-range magnetic ordering is frustrated by the geometry of the crystalline lattice have attracted much attention recently, since the geometrical magnetic frustration results in the existence of many energetically equivalent magnetic ground states [37-39]. So far, magnetic frustra- 


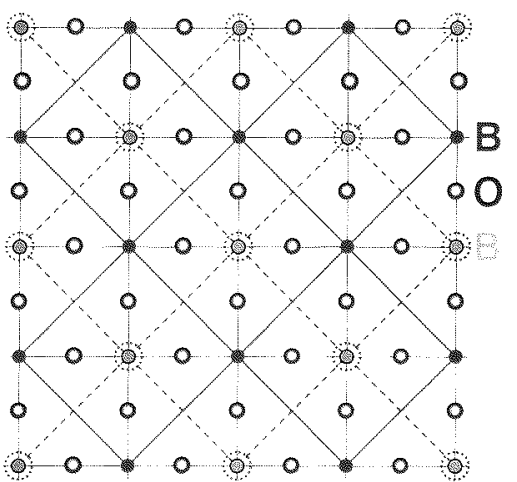

(a)

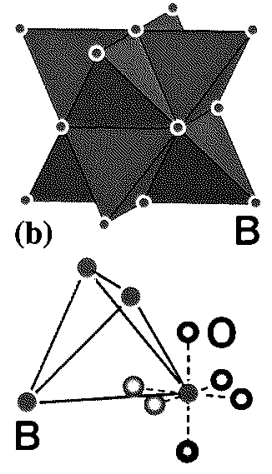

(c)

Fig. 2. The atom positions and magnetic sublattice in double perovskites with $\mathrm{B}=$ magnetic lanthanide ion. In part (a) the positions of the magnetic B-site ions in the second plane or marked by dotted circles. The thick lines indicate the near neighbor magnetic interactions within the planes. In part (b) the 3D arrangement of the edge-shared magnetic ion tetrahedra is shown. Part (c) shows the B-O coordination polyhedra.

tion has been studied in 2D Kagomé lattice systems consisting of corner-shared triangles of magnetic ions. The $3 \mathrm{D}$ extension is a corner-sharing tetrahedron lattice realized in the magnetic lanthanide pyrochlores [40, 41]. Perfectly ordered double perovskites have magnetic sublattices consisting of edge-sharing tetrahedra and represent another frustrating geometry in three dimensions. So far, reports on the magnetic properties of these highly interesting magnetic systems are rare [36].

\subsection{Double perovskites with $B=$ magnetic transition metal ion}

In double perovskite systems $\mathrm{A}_{2} \mathrm{BB}^{\prime} \mathrm{O}_{6}$ with $\mathrm{B}$ a magnetic transition metal ion with less localized $3 d$ or $4 d$ states, the magnetic exchange is dominated by hopping of electrons between the different sites. These processes can result both in ferromagnetic or antiferromagnetic coupling. Antiferromagnetic superexchange via the $\mathrm{B}-\mathrm{O}-\mathrm{B}^{\prime}-\mathrm{O}-\mathrm{B}$ exchange path is usually dominant for $\mathrm{B}^{\prime}$ ions with noble gas configuration such as $\mathrm{Nb}^{5+}$ or $\mathrm{W}^{6+}$. Ferromagnetic exchange mechanisms (double exchange type [42-44] or hybridization type mechanism [45-47]) are dominant for $\mathrm{B}^{\prime}$ ions with delocalized $4 d$ or $5 d$ electrons such as $\mathrm{Mo}^{5+}, \mathrm{W}^{5+}, \mathrm{Re}^{5+}$, or $\mathrm{Re}^{6+}$. The hybridization between the delocalized $\mathrm{B}^{\prime}$ and the localized $\mathrm{B}$ states usually results in a kinetic energy driven mechanism causing a strong ferromagnetic exchange between the B-sites.

\section{D.1. Antiferromagnetic superexchange}

The situation for the antiferromagnetic superexchange via the $\mathrm{B}-\mathrm{O}-\mathrm{B}^{\prime}-\mathrm{O}-\mathrm{B}$ exchange path is sketched in Fig. 3. This exchange mechanism is dominant for $\mathrm{B}^{\prime}$ ions with noble gas configuration such as $\mathrm{Nb}^{5+}$ or $\mathrm{W}^{6+}$, i.e. a completely empty $4 d$ - or $5 d$-band. Using the simple rule that there is an antiferromagnetic exchange 

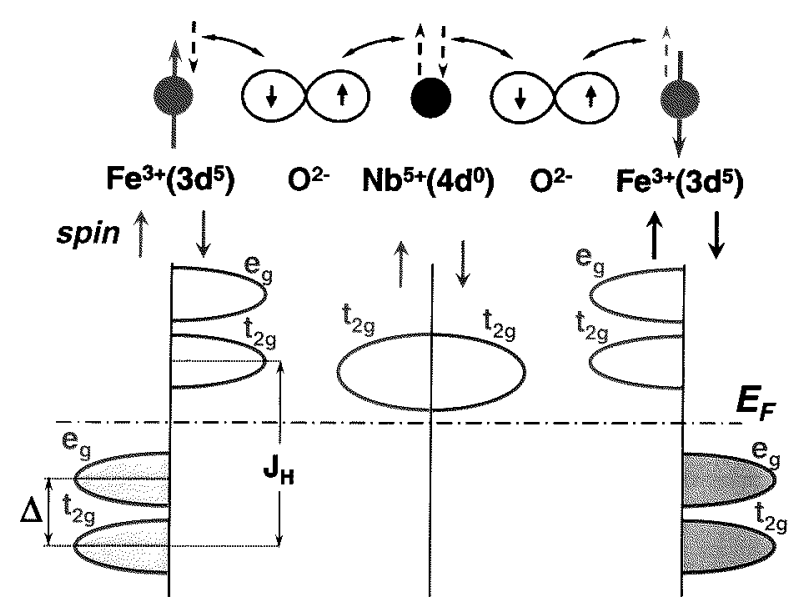

Fig. 3. Superexchange mechanism via the $\mathrm{B}-\mathrm{O}-\mathrm{B}^{\prime}-\mathrm{O}-\mathrm{B}$ exchange path in double perovskites, where the $\mathrm{B}^{\prime}$ has a completely empty d-shell. The superexchange causes an antiferromagnetic configuration between the localized Fe $3 d \uparrow$ spins. The thick arrow mark the $\mathrm{Fe} 3 d^{5}$ core spins, the broken arrows indicate the spin configuration of an excited state. The lower part shows the situation in a band-like picture for the two spin directions. Here, $\Delta$ denotes the crystal field splitting between the $t_{2 \mathrm{~g}}$ and the $e_{\mathrm{g}}$ band, $J_{\mathrm{H}}$ the exchange splitting. The $\mathrm{Nb} e_{\mathrm{g}}$ band is well above the $t_{2 \mathrm{~g}}$ band and not shown.

between two empty orbitals (e.g. between empty $\mathrm{Fe} 3 d \downarrow / \mathrm{Fe} 3 d \uparrow$ and the $\mathrm{Nb} 4 d$ orbitals as sketched in Fig. 3), we obtain an antiferromagnetic coupling between the B-sites. In a band-like picture, both the $\mathrm{Fe} t_{2 \mathrm{~g}} \downarrow$ and the $\mathrm{Nb} t_{2 \mathrm{~g}}$ states are empty.

In general, for the double perovskites the antiferromagnetic superexchange is weak due to the long exchange path and therefore results in low Néel temperatures $T_{\mathrm{N}}$ of the order of a few $10 \mathrm{~K}$. Typical examples are $\mathrm{Sr}_{2} \mathrm{FeNbO}_{6}$ with a Néel temperature of about $25 \mathrm{~K}[24-26], \mathrm{Ca}_{2} \mathrm{CoWO}_{6}$ and $\mathrm{Ca}_{2} \mathrm{NiWO}_{6}$ with $T_{\mathrm{N}}=26$ and $56 \mathrm{~K}$, respectively [30], as well as $\mathrm{Sr}_{2} \mathrm{FeWO}_{6}$ with $T_{\mathrm{N}}=37 \mathrm{~K}$ [27-29]. We also note that the exchange strength depends on the bonding angles along the exchange path and usually is maximum for a $180^{\circ}$ superexchange. Furthermore, disorder on the $\mathrm{BB}^{\prime}$ sublattices can result in spin-glass-like behavior.

On electron doping the system by replacing e.g. $\mathrm{Sr}^{2+}$ by $\mathrm{La}^{3+}$ in $\mathrm{Sr}_{2} \mathrm{FeNbO}_{6}$ the Fermi level shifts into the $\mathrm{Nb} 4 d t_{2 \mathrm{~g}}$ band. In this way mobile electrons are available on the $\mathrm{B}^{\prime}$-site favoring a ferromagnetic interaction as discussed below. At low doping, a competition between antiferromagnetic super- and ferromagnetic double exchange occurs which may result in a spin-glass state.

\section{2. . Ferromagnetic double exchange}

One of the puzzling features of magnetic double perovskites is the strong ferromagnetic exchange with Curie temperatures up to $635 \mathrm{~K}[7]$ in systems, where we 
have non-magnetic $\mathrm{B}^{\prime}$ ions with delocalized electrons. The origin of ferromagnetism in these double perovskites is still discussed controversially. Recently Moritomo et al. found a strong correlation between the room temperature conductivity and the Curie temperature in $\mathrm{Sr}_{2} \mathrm{FeMoO}_{6}$, implying that the mobile conduction electrons mediate the exchange interaction between the local $\mathrm{Fe}^{3+}$ spins $[42,43]$. Therefore, in analogy to the doped manganites it was tempting to explain the ferromagnetic coupling between the Fe sites based on a double exchange mechanism, where the delocalized $t_{2 \mathrm{~g}}$ electron provided by the Mo $4 d^{1}$ configuration plays the role of the delocalized $\mathrm{Mn} 3 d e_{\mathrm{g}}$ electron in the manganites.

The situation for the double exchange type exchange mechanism is sketched in Fig. 4 using the example of $\mathrm{Sr}_{2} \mathrm{FeMoO}_{6}$. If the Fe $t_{2 \mathrm{~g}} \downarrow$ and the Mo $t_{2 \mathrm{~g}} \downarrow$ bands are almost degenerate (this is equivalent to degenerate valence states $\mathrm{Fe}^{3+}-\mathrm{Mo}^{5+}$ and $\mathrm{Fe}^{2+}-\mathrm{Mo}^{6+}$ (see the top part of Fig. 4), the $t_{2 \mathrm{~g}}$ electron can easily hop between these states producing a double exchange type interaction [48, 49]. That is, hopping of itinerant spin-down electrons between the Fe and Mo sites yields a kinetic energy gain. Since the Fe $3 d \uparrow$ band is completely filled, this hopping of the $t_{2 \mathrm{~g}}$ electrons is only possible, if the Fe core spins are all oriented antiparallel to the spins of the itinerant electrons. As a consequence, a ferromagnetic state with all Fe spins parallel will be energetically favored. The Mo $4 d t_{2 \mathrm{~g}} \uparrow$ electrons cannot hop in this

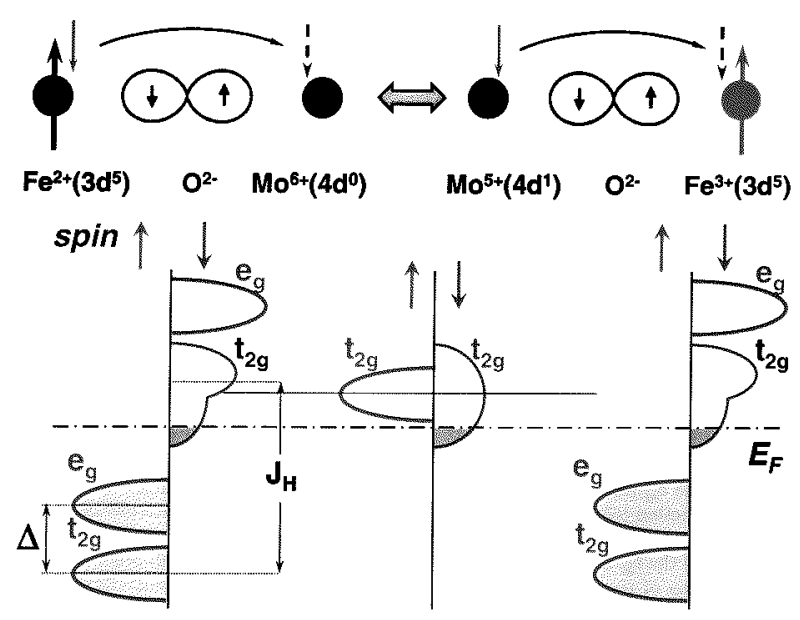

Fig. 4. Double exchange type exchange mechanism in double perovskites, where the $\mathrm{B}^{\prime}$ has a partly filled $d$-shell. The two configurations shown in the top part are degenerate. The thick arrows mark the localized Fe $3 d^{5}$ core spins, the thin arrows the spin of the itinerant electron. The bottom part shows the band picture. The Fe $t_{2 \mathrm{~g}} \downarrow$ and the Mo $t_{2 \mathrm{~g}} \downarrow$ bands are almost degenerate resulting in degenerate valence states $\mathrm{Fe}^{3+}-\mathrm{Mo}^{5+}$ and $\mathrm{Fe}^{2+}-\mathrm{Mo}^{6+}$. The hopping of the $3 d t_{2 \mathrm{~g}} \downarrow$ electrons results in a broadening of the Mo $4 d t_{2 \mathrm{~g}}$ and Fe $3 d t_{2 \mathrm{~g}} \downarrow$ bands. 
situation. Therefore, only the Mo $4 d t_{2 \mathrm{~g}} \downarrow$ is broadened resulting in a half-metallic behavior (see the bottom part of Fig. 4).

We note that the situation in the double perovskites is different to the double exchange in colossal magnetoresistance (CMR) manganites, where the itinerant $e_{\mathrm{g}} \uparrow$ spins are parallel to the localized $t_{2 \mathrm{~g}} \uparrow$ core spins. In the double perovskites the completely full $\mathrm{Fe} 3 d \uparrow$ band makes it impossible for another spin-up electron to hop between Fe sites and thus forcing the delocalized electrons to be spin-down electrons. Moreover, in the manganites both the localized $\mathrm{Mn} t_{2 \mathrm{~g}}$ electrons and the delocalized $\mathrm{Mn} e_{\mathrm{g}}$ electron reside at the same site and their spins are coupled ferromagnetically by a strong on-site Hund coupling $J_{\mathrm{H}}$. In the double perovskites, the localized Fe $3 d$ electrons ( $\left.\mathrm{Fe}^{3+}: 3 d^{5}, S=5 / 2\right)$ and the delocalized Mo $4 d$ electron $\left(\mathrm{Mo}^{5+}: 4 d^{1}, S=1 / 2\right)$ nominally are at two different sites although the Mo $4 d$ electron obtains a finite Fe character by sizable hopping interaction. Therefore, at first glance the double exchange scenario is different in the manganites and the double perovskites.

If the B-site $t_{2 \mathrm{~g}} \downarrow$ and the $\mathrm{B}^{\prime}$-site $t_{2 \mathrm{~g}} \downarrow$ bands are not degenerate, as it is the case for the system $\mathrm{Sr}_{2} \mathrm{MnMoO}_{6}[50,51]$, the hopping is very weak and, hence, a much weaker ferromagnetic interaction is expected within a double exchange type model.

\subsubsection{Kinetic energy driven ferromagnetic exchange}

Sarma $[9,45]$ has proposed another mechanism to explain ferromagnetism in the double perovskites, which has been extended to many other systems by Fang, Kanamori, and Terakura [46, 47]. In the Sarma-Fang-Kanamori-Terakura (SFKT) model, the hybridization of the Mo $4 d\left(t_{2 \mathrm{~g}}\right)$ and Fe $3 d\left(t_{2 \mathrm{~g}}\right)$ states plays the key role in stabilizing ferromagnetism at high Curie temperatures [45-47]. The essence of this model is summarized in Fig. 5 for the case of $\mathrm{Sr}_{2} \mathrm{FeMoO}_{6}$. Without any hopping interactions, the $\mathrm{Fe}^{3+} 3 d^{5}$ configuration has a large exchange splitting of the $3 d$ levels in the spin-up and spin-down states and there is also a crystal field splitting $\Delta$ into the $t_{2 \mathrm{~g}}$ and the $e_{\mathrm{g}}$ states. The exchange splitting of the $\mathrm{Mo}^{5+} 4 d^{1}$ configuration (better the Mo- $4 d-\mathrm{O}-2 p$ hybridized states) is vanishingly small, however, there is a large crystal field splitting (the $e_{\mathrm{g}}$ states are several $\mathrm{eV}$ above the $t_{2 \mathrm{~g}}$ states and not shown in Fig. 5). Switching on the hopping interactions results in a finite coupling between states of the same symmetry and spin. The hopping interaction not only leads to an admixture of the Fe $3 d$ to the Mo $4 d$ states, but more importantly to a shift of the bare energy levels. Asshown in Fig. 5, the delocalized Mo $t_{2 \mathrm{~g}}$ spin-up states are pushed up, whereas the Mo $t_{2 \mathrm{~g}}$ spin-down states are pushed down (bonding-antibonding splitting). This causes a finite spin polarization at the Fermi level (actually $100 \%$ in Fig. 5) resulting from the hopping interactions. It is evident that this mechanism leads to an antiferromagnetic coupling between the delocalized Mo $4 d$ and the localized Fe $3 d$ electrons, since the energy is lowered by populating the Mo $4 d$ spin-down band [45]. The magnitude 


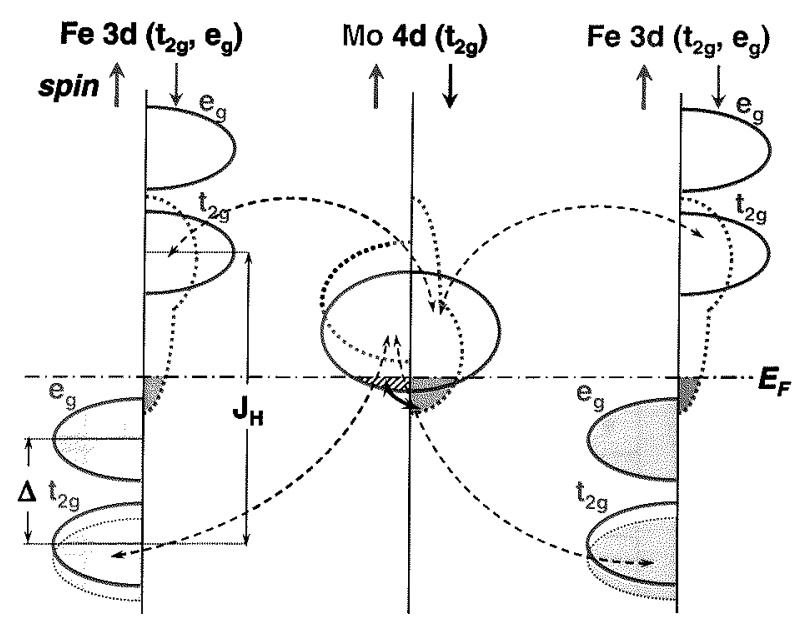

Fig. 5. Sketch of the band structure for the illustration of the mechanism stabilizing the ferromagnetic state in the double perovskites using $\mathrm{A}_{2} \mathrm{FeMoO}_{6}$ as an example. The Fermi energy lies between the exchange split $3 d$ spin-up and -down states. The solid lines mark the bands without hybridization, whereas the broken lines mark the bands with hybridization. The broken arrows connect the hybridizing bands. The hybridization between the $e_{\mathrm{g}}$ level has been neglected. The Mo $4 d e_{\mathrm{g}}$ band is not shown.

of the spin polarization derived from this mechanism obviously is governed by the hopping strength and the charge transfer energy between the localized and the delocalized states [46]. Furthermore, the itinerant electrons in the Mo $t_{2 \mathrm{~g}} \downarrow$ band cause kinetic energy gain via a double exchange type interaction, which further stabilizes ferromagnetism as discussed above. In view of kinetic energy gain the SFKT model is similar to the double exchange model discussed above.

The essential point in the SFKT scenario is that the hybridized states are located energetically between the exchange-split Fe $3 d \uparrow$ and the Fe $3 d \downarrow$ levels. Only in this case a bonding-antibonding splitting is obtained. We also note that in the SFKT model the magnetic moment at the Mo site is merely induced by the Fe magnetic moments through the hybridization between the $\mathrm{Fe} 3 d$ and the Mo $4 d$ states which can be viewed as a magnetic proximity effect. In this sense the double perovskites should be denoted ferromagnetic and not ferrimagnetic. Recently, it has been shown that the magnetism in $\mathrm{Sr}_{2} \mathrm{FeMoO}_{6}$ can be well described by two interacting sublattices: the localized $\mathrm{Fe}^{3+} 3 d^{5}$ core and the delocalized $\mathrm{Mo}^{5+} 4 d^{1}$ delocalized electron spins with a strong antiferromagnetic interaction between the two sublattices in good agreement with the SFKT model [52].

We have pointed out recently [14] that the mechanism discussed above also works for the $\mathrm{A}_{2} \mathrm{CrWO}_{6}$ system, where only the $\mathrm{Cr} 3 d t_{2 \mathrm{~g}} \uparrow$ band is full, however, the $\mathrm{Cr} 3 d e_{\mathrm{g}} \uparrow$ band completely empty. The key concept of the model again is the energy gain contributed by the spin polarization of the non-magnetic ele- 
ment (now W) induced by the hybridization with the magnetic transition metal (now Cr). It has been pointed out by Sarma et al. [9, 45] that the underlying mechanism will always be operative, whenever the conduction band is placed within the energy gap formed by the large exchange splitting of the localized electrons at the transition metal site. As shown in Fig. 6, this is also the case for the $\mathrm{A}_{2} \mathrm{CrWO}_{6}$ compounds: the $\mathrm{W} 5 d t_{2 \mathrm{~g}}$ band resides in between the exchange split $\mathrm{Cr} 3 d t_{2 \mathrm{~g}}$ spin-up and spin-down bands. The schematic band structure of Fig. 6 has been confirmed by band structure calculations [14]. The only difference between the system $\mathrm{AsCrWO}_{6}$ and the systems $\mathrm{A}_{2} \mathrm{FeMoO}$ (or also $\mathrm{A}_{2} \mathrm{FeReO}_{6}$ ) is the fact that in the former the majority spin band is only partly full. Furthermore, band structure calculations show that the crystal field splitting in the Cr compounds $(\approx 2 \mathrm{eV})$ is slightly larger than in the Fe compounds [53]. On the other hand, the exchange splitting in the Cr $3 d$ bands is somewhat smaller than for the Fe $3 d$ bands due to the valence configuration $\mathrm{Cr} 3 d^{3}$ with less electrons and weaker Hund's coupling. Taking these facts into account we have to split up the Cr $3 d$ spin-up and spin-down band into two separate $3 d t_{2 \mathrm{~g}}$ and $3 d e_{\mathrm{g}}$ bands with the Fermi level lying in the gap between the bands as shown in Fig. 6. Indeed, band structure calculations [14] show that the $\mathrm{Cr} 3 d e_{\mathrm{g}}$ spin-up band is about $0.5 \mathrm{eV}$ above the Fermi level. However, the above mechanism still works as long as the

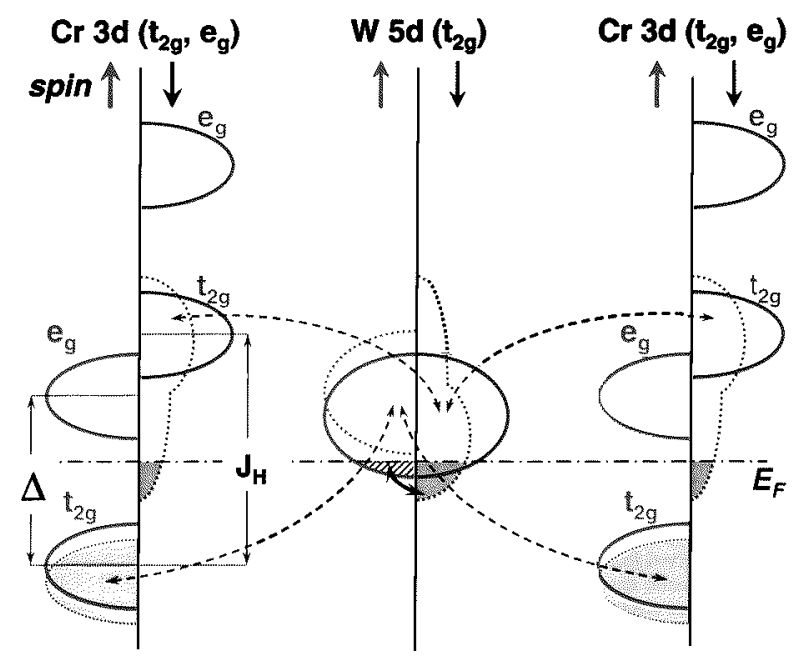

Fig. 6. Sketch of the band structure for the illustration of the mechanism stabilizing the ferromagnetic state in the double perovskites using $\mathrm{A}_{2} \mathrm{CrWO}_{6}$ as an example. In contrast to the $\mathrm{A}_{2} \mathrm{FeMoO}_{6}$ system the Fermi energy lies between the crystal field split $3 d t_{2 \mathrm{~g}} \uparrow$ and $3 d e_{\mathrm{g}} \uparrow$ states. The solid lines mark the bands without hybridization, whereas the broken lines mark the bands with hybridization. The broken arrows connect the hybridizing bands. The hybridization between the $e_{\mathrm{g}}$ level has been neglected. The W $5 d e_{\mathrm{g}}$ band is not shown. 
W $5 d t_{2 \mathrm{~g}}$ band is placed within the energy gap between the $\mathrm{Cr} 3 d t_{2 \mathrm{~g}}$ spin-up and the $\mathrm{Cr} 3 d t_{2 \mathrm{~g}}$ spin-down band. Then, again the $\mathrm{W} 5 d t_{2 \mathrm{~g}}$ levels hybridize with the Cr $3 d t_{2 \mathrm{~g}}$ levels resulting in a negative spin polarization of the non-magnetic element $\mathrm{W} 5 d t_{2 \mathrm{~g}}$ and a stabilization of ferromagnetism and half-metallic behavior. We note that no hybridization takes place between the $\mathrm{Cr} 3 d e_{\mathrm{g}}$ spin-up band and the $W 5 d t_{2 \mathrm{~g}}$ spin-up band due to the different symmetry of these levels. Therefore, the exact position of the $\mathrm{Cr} 3 d e_{\mathrm{g}}$ spin-up band is not relevant. Summarizing our discussion we can state that within the SFKT model the essential physical mechanism leading to ferromagnetism is very similar for the $\mathrm{A}_{2} \mathrm{CrWO}_{6}$ and the $\mathrm{A}_{2} \mathrm{FeMoO}_{6}$ compounds.

\section{The double perovskite $\mathrm{A}_{2} \mathrm{CrWO}_{6}$}

In this section we discuss our experimental results on bulk samples as well as epitaxial films of the double perovskite system $\mathrm{A}_{2} \mathrm{CrWO}_{6}$ with $\mathrm{A}=\mathrm{Sr}, \mathrm{Ca}, \mathrm{Ba}$. The sample fabrication has been discussed already recently $[12,14]$.

\subsection{Structural properties}

The structure of the system $\mathrm{A}_{2} \mathrm{CrWO}_{6}$ is determined by the tolerance factor $f$, which is varying with varying ionic radius of the A-site cation. The crystal structure of the different compounds was determined by a Rietveld refinement of the X-ray data [14]. While $\mathrm{Sr}_{2} \mathrm{Cr} \mathrm{WO}_{6}$ has an almost ideal tolerance factor $f \simeq 1$ resulting in a cubic structure $(F m \overline{3} m)$, for $\mathrm{Ca}_{2} \mathrm{CrWO}_{6}$ the tolerance factor is much smaller $(f=0.945)$ resulting in a monoclinic system $\left(P 2_{1} / n\right)$. For $\mathrm{Ba}_{2} \mathrm{CrWO}_{6}$, the tolerance factor is much larger than $1(f=1.059)$ resulting in a 6-layered hexagonal structure $(P \overline{6} 2 c)$.

Comparing the crystallographic properties of the $\mathrm{A}_{2} \mathrm{CrWO}_{6}$ systems to those of other double perovskites [54-57] shows that cubic or tetragonal structures are obtained only for tolerance factors in the range between $0.96 \lesssim f \lesssim 1.06$. For $f \lesssim 0.96$, orthorhombic/monoclinic structures are favored, whereas for $1.06 \lesssim f$ a hexagonal structure is preferred (see Fig. 7). As will be shown below, for most double perovskites a maximum $T_{\mathrm{C}}$ is obtained for a tolerance factor of $f \simeq 1$ corresponding to an about cubic perovskite structure with a $\mathrm{O}-\mathrm{B}-\mathrm{O}$ bond angle close to $180^{\circ}$. The requirement $f \simeq 1$ for optimum $T_{\mathrm{C}}$ in the double perovskite is different for the doped manganites. Here, a maximum $T_{\mathrm{C}}$ is achieved for compounds with $f \lesssim 0.95$, that is, for a significantly distorted perovskite structure.

Hwang et al. [58] have shown that the highest $T_{\mathrm{C}}$ in doped manganites is obtained for $f \simeq 0.93$ (in a more precise analysis by Zhou et al. [59] slightly larger values for $f$ have been derived based on a coordination number of 9 ). A further difference between the doped manganites and the double perovskite is related to the size of the A-site cations. In the former we usually have a significant variance of the ionic radii of the A-site cations, whereas this is not the case in the double perovskites 
with $\mathrm{A}_{2}=\mathrm{Sr}$, Ca, Ba. Of course, it is tempting to state that the different behavior of the manganites and the double perovskites is related to differences in the underlying exchange mechanisms. However, a definite answer regarding this issue cannot yet be given.

We also have analyzed the $\mathrm{Cr} / \mathrm{W}$ sublattice order for $\mathrm{A}_{2} \mathrm{CrWO}_{6}$. For the $\mathrm{Sr}$ system the amount of antisites is $23 \%$, for the Ca system only $13 \%$. We first note that the antisite density for $\mathrm{A}_{2} \mathrm{CrWO}_{6}$ is considerably larger than for $\mathrm{A}_{2} \mathrm{FeMoO}_{6}$. This is caused by the very similar ionic radii of the $\mathrm{Cr}\left(r_{\mathrm{Cr}^{3+}}=0.615 \AA\right)$ and $\mathrm{W}$ ions $\left(r_{\mathrm{W}^{5+}}=0.62 \AA\right)$ compared to the significantly different radii of $\mathrm{Fe}\left(r_{\mathrm{Fe}^{3+} \text {, high spin }}=\right.$ $0.645 \AA)$ and Mo ions $\left(r_{\mathrm{Mo}^{5+}}=0.61 \AA\right)$. Second, the lower amount of antisites in $\mathrm{Ca}_{2} \mathrm{Cr} \mathrm{WO}_{6}$ may be associated with a gradual transition from $\mathrm{W}^{5+}$ to $\mathrm{W}^{6+}$ $\left(r_{\mathrm{Cr}^{2+}}=0.73 \AA\right.$ and $\left.r_{\mathrm{W}^{6+}}=0.60 \AA\right)$. Due to its hexagonal structure the situation for the $\mathrm{Ba}_{2} \mathrm{CrWO}_{6}$ compound is more difficult [14].

\subsection{Magnetic properties}

The saturation magnetization in $\mathrm{A}_{2} \mathrm{CrWO}_{6}$ measured at $5 \mathrm{~K}$ was found to decrease with increasing amount $\delta$ of antisites ( $\delta=0.5$ for complete disorder) in agreement with other studies $[16,20]$. This can be understood in terms of the SFKT model by assuming that there is an antiferromagnetic coupling of two sublattices formed by the ferromagnetically ordered $\mathrm{Cr}^{3+} 3 d^{3}$ core spins and the delocalized $\mathrm{W}^{5+} 5 d^{1}$ delocalized electron spins. In the presence of antisite defects, there are also neighboring $\mathrm{Cr}$ ions coupling antiferromagnetically via the superexchange. Then, the saturation magnetization $M_{\text {sat }}$ is expected to follow the expression

$$
M_{\text {sat }}(\delta)=(1-2 \delta) m\left(\mathrm{Cr}^{3+}\right)-(1-2 \delta) m\left(\mathrm{~W}^{5+}\right),
$$

where $m\left(\mathrm{Cr}^{3+}\right)$ and $m\left(\mathrm{~W}^{5+}\right)$ are the magnetic moments of the $\mathrm{Cr}^{3+}$ and $\mathrm{W}^{5+}$ ions in units of $\mu_{\mathrm{B}}$, respectively. Our experimental results are in reasonable agreement with this expectation. For $\mathrm{Sr}_{2} \mathrm{CrWO}_{6}$, we observe $M_{\text {sat }}=1.11 \mu_{\mathrm{B}} /$ f.u. very close to the expected value of $1.08 \mu_{\mathrm{B}} / \mathrm{f}$.u. for $\delta=0.23$. For $\mathrm{Ca}_{2} \mathrm{CrWO}_{6}$ we have $\delta=0.13$ and hence expect $M_{\text {sat }}=1.48 \mu_{\mathrm{B}} / \mathrm{f}$.u. This value is slightly larger than the measured value of $M_{\text {sat }}=1.34 \mu_{\mathrm{B}} /$ f.u., most likely due to the distorted crystal structure of $\mathrm{Ca}_{2} \mathrm{CrWO}_{6}$. A detailed quantitative analysis is not possible at present since a theoretical prediction for $M_{\text {sat }}(\delta)$ based on the SFKT model has not yet been worked out.

We next discuss the Curie temperature. We found that the substitution of $\mathrm{Sr}$ by $\mathrm{Ca}$ or $\mathrm{Ba}$ in $\mathrm{A}_{2} \mathrm{CrWO}_{6}$ results in a strong reduction of $T_{\mathrm{C}}$ from $458 \mathrm{~K}(\mathrm{Sr})$ to $161 \mathrm{~K}(\mathrm{Ca})$ and $141 \mathrm{~K}(\mathrm{Ba})$. The important point is that the compound with a tolerance factor $f \simeq 1$ has the maximum $T_{\mathrm{C}}$. In particular, the magnetic properties of the $\mathrm{Ba}_{2} \mathrm{CrWO}_{6}$ compound are completely different from those of $\mathrm{Sr}_{2} \mathrm{CrWO}_{6}$ and $\mathrm{Ca}_{2} \mathrm{CrWO}_{6}$. Here, the large ionic radius of $\mathrm{Ba}^{2+}$ enlarges $f$ well above unity. This causes a structural phase transition towards a hexagonal structure, where the ferromagnetic interaction is strongly suppressed [14]. 
Comparing our results for the series $\mathrm{A}_{2} \mathrm{CrWO}_{6}$ to other double perovskite compounds (see Fig. 7), it becomes evident that the suppression of $T_{\mathrm{C}}$ as a function of the deviation of the tolerance factor from its ideal value of $f=1$ is a general trend: it is only weak for the series $\mathrm{A}_{2} \mathrm{Fe} \mathrm{MoO}_{6}$ with $\mathrm{A}=\mathrm{Sr}, \mathrm{Ba}$, Ca, where $T_{\mathrm{C}}$ varies between $310 \mathrm{~K}$ and $420 \mathrm{~K}[1,54-57]$. However, it is also strong for the series $\mathrm{A}_{2} \mathrm{CrReO}_{6}$ with $T_{\mathrm{C}}=635 \mathrm{~K}$ for $\mathrm{Sr}_{2} \mathrm{CrReO}_{6}$ and $T_{\mathrm{C}}=360 \mathrm{~K}$ for $\mathrm{Ca}_{2} \mathrm{CrReO}_{6}[7]$. In general, a high Curie temperature can only be realized in double perovskites of the composition $\mathrm{A}_{2} \mathrm{BB}^{\prime} \mathrm{O}_{6}$ having a tolerance factor close to unity. This is realized in the different systems for $\mathrm{A}_{2}=\mathrm{Sr}_{2}$. For $f$ well below unity, the Curie temperature is drastically reduced in agreement with what is found for the doped manganites [58]. For the double perovskites, the system $\left(\mathrm{Sr}_{1-y} \mathrm{Ca}_{y}\right)_{2} \mathrm{FeReO}_{6}$ is an exception of the general rule [60]: here, the $\mathrm{Ca}_{2} \mathrm{FeReO}_{6}$ compound has the highest $T_{\mathrm{C}}$, although the tolerance factor decreases continuously from $f=0.997$ for $\mathrm{Sr}_{2} \mathrm{FeReO}_{6}$ to $f=0.943$ for $\mathrm{Ca}_{2} \mathrm{FeReO}_{6}$ on substituting $\mathrm{Sr}$ by $\mathrm{Ca}$. We note, however, that $\mathrm{CaFeReO}_{6}$ is a unique material as it is a ferromagnetic insulator and that there may be an other mechanism causing the high ordering temperature [23].

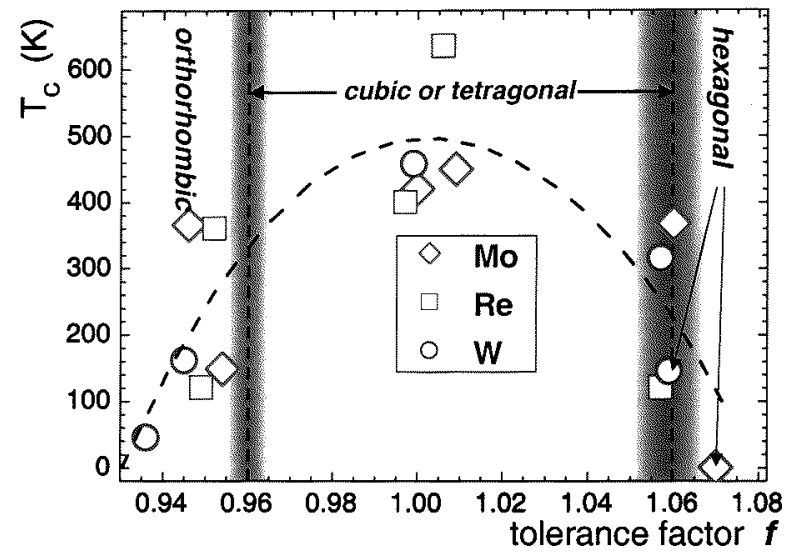

Fig. 7. Curie temperatures of the different double perovskite materials of this study completed by data taken from literature plotted versus their tolerance factor. Re-compounds: $\square, \mathrm{W}$-compounds: $\circ$, Mo-compounds: $\diamond$. The broken line only serves as a guide to the eyes. The shaded areas indicate that the transition between the regions with different structures are not sharp.

We briefly discuss the observed variation of $T_{\mathrm{C}}$ with varying tolerance factor in the context of the models discussed above. It is well known that first the tolerance factor is intimately related to the bond angles and second that in perovskite type transition metal oxides in general the increase in the $\mathrm{B}-\mathrm{O}$ bond length and the deviation of the $\mathrm{B}-\mathrm{O}-\mathrm{B}$ bond angle from $180^{\circ}$ has the effect of reducing the hopping amplitude $t$. This is caused by the reduction of the overlap of the oxygen 
$2 p$ and transition-metal $d$ states [61]. Since the weakening of the hopping causes a reduction of the energy gain stabilizing ferromagnetism both within a double exchange type and the SFKT model, we expect a significant decrease in $T_{\mathrm{C}}$ with increasing deviation of the tolerance factor from $f=1$ or, equivalently, with an increasing deviation of the $\mathrm{B}-\mathrm{O}-\mathrm{B}$ bond angle from $180^{\circ}$. This is in good qualitative agreement with our results on the $\mathrm{A}_{2} \mathrm{CrWO}_{6}$ compound and the collected data plotted in Fig. 7. Theoretical models providing a quantitative explanation have still to be developed.

\subsection{Magnetotransport properties}

Figure 8 shows the temperature dependence of the resistivity $\rho$ for the double perovskite system $\mathrm{A}_{2} \mathrm{CrWO}_{6}$ with $\mathrm{A}=\mathrm{Sr}, \mathrm{Ba}$, Ca. All samples show an increase in the resistivity with decreasing temperature. Since the investigated samples are polycrystalline, the influence of grain boundaries plays an important role. Hence, the observed semiconductor-like $\rho(T)$ curves most likely are related to the grain boundary resistance, whereas the intrinsic resistance is metallic. We also studied the $\rho(T)$ curves of epitaxial thin films containing no grain boundaries. Although these films show metallic behavior, the intrinsic resistivity behavior of the thin films could not be derived unambiguously, since the surface of the $\mathrm{SrTiO}_{3}$ substrate becomes conducting under the deposition conditions [13].

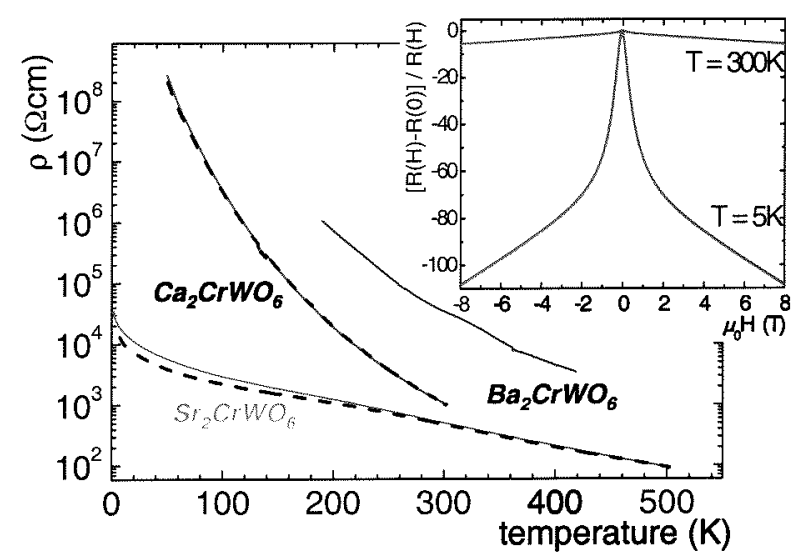

Fig. 8. Resistivity vs. temperature for the double perovskites $\mathrm{A}_{2} \mathrm{CrWO}_{6}$ with $\mathrm{A}=\mathrm{Sr}$, $\mathrm{Ba}, \mathrm{Ca}$. The dashed lines are taken at an applied field of $80 \mathrm{kOe}$. The inset shows the magnetoresistance vs. applied magnetic field curve of $\mathrm{Sr}_{2} \mathrm{CrWO}_{6}$ at $T=5$ and $300 \mathrm{~K}$.

Although the intrinsic resistivity behavior could not be unambiguously derived from our measurements, the fact that $\mathrm{d} \ln \sigma / \mathrm{d} \ln T \rightarrow 0$ for $T \rightarrow 0$ [14] provides significant evidence for a metallic behavior in the $\mathrm{Sr}_{2} \mathrm{CrWO}_{6}$ sample. Here, $\sigma$ is the electrical conductivity. The observed trend that the resistivity increases, if $\mathrm{Sr}_{2}$ is replaced by $\mathrm{Ca}_{2}$ and even more by $\mathrm{Ba}_{2}$, can be understood as follows: in contrast 
to the cubic perovskite $\mathrm{Sr}_{2} \mathrm{CrWO}_{6}, \mathrm{Ca}_{2} \mathrm{CrWO}_{6}$ has a distorted perovskite structure with a $\mathrm{B}-\mathrm{O}-\mathrm{B}$ bonding angle deviating from $180^{\circ}$. This results in a reduction of the overlap between the relevant orbitals and, hence, the hopping amplitude. Finally, the $\mathrm{Ba}_{2} \mathrm{CrWO}_{6}$ compound has the highest resistivity most likely due to its hexagonal structure.

The dashed curves in Fig. 8 show the $\rho(T)$ curves measured at an applied field of $80 \mathrm{kOe}$. It is evident that the largest magnetoresistance is observed for the $\mathrm{Sr}_{2} \mathrm{CrWO}_{6}$ sample. As shown in the inset, the polycrystalline $\mathrm{Sr}_{2} \mathrm{CrWO}_{6}$ sample containing a large number of grain boundaries shows a large negative low-field magnetoresistance, $\mathrm{MR}=[R(H)-R(0)] / R(H)$, of up to about $-100 \%$ at $5 \mathrm{~K}$ and $8 \mathrm{~T}$. At room temperature, this effect is reduced to a few percent. The large grain boundary MR effect at low temperatures indicates that $\mathrm{Sr}_{2} \mathrm{CrWO}_{6}$ has a large spin polarization of the charge carriers and due to its high Curie temperature may be an interesting candidate for spintronic devices operating at room temperature.

\subsection{Optical properties}

We have performed optical reflection and transmission measurements of $\mathrm{Sr}_{2} \mathrm{CrWO}_{6}$ thin films with photon energies from $0.38 \mathrm{eV}$ to $7 \mathrm{eV}$ [14]. Since the $\mathrm{SrTiO}_{3}$ substrates, on which the epitaxial quality of the films is high, are transparent only for photon energies in the range $0.20-3.2 \mathrm{eV}$, we have also investigated strained epitaxial $\mathrm{Sr}_{2} \mathrm{CrWO}_{6}$ films on $\mathrm{LaAlO}_{3}$ substrates, which are transparent from 0.17 to $5.5 \mathrm{eV}$, and polycrystalline $\mathrm{Sr}_{2} \mathrm{CrWO}_{6}$ films on $\mathrm{MgAl}_{2} \mathrm{O}_{3}$ substrates, which are transparent between $0.22-6.5 \mathrm{eV}$. The optical measurements show an increase in the absorption coefficient of $\mathrm{Sr}_{2} \mathrm{CrWO}_{6}$ above $4 \mathrm{eV}$, which can be attributed to a charge transfer transition between the $p$-like spin-up and -down oxygen bands at $-3 \mathrm{eV}$ below the Fermi level into the oxygen/metal bands at $+1 \mathrm{eV}$ above the Fermi level. Furthermore, an absorption shoulder around $1 \mathrm{eV}$ was found, which coincides roughly with the energy gap at the Fermi level in the spin-up band, and is therefore probably caused by transitions from the Cr spin-up $t_{2 \mathrm{~g}}$ states below the Fermi level into the oxygen/metal bands around $+1 \mathrm{eV}$ above the Fermi level. Unfortunately, due to the substrate absorption below $0.2 \mathrm{eV}$, no optical information is available in the infrared region which would allow a more definitive statement with respect to the density of states at the Fermi level and the half-metallic character of $\mathrm{Sr}_{2} \mathrm{CrWO}_{6}$.

\subsection{Electron doping}

We have studied the effect of electron doping in $\mathrm{Sr}_{2} \mathrm{CrWO}_{6}$ in a series of $\mathrm{Sr}_{2-x} \mathrm{La}_{x} \mathrm{CrWO}_{6}$ samples with $x=0,0.1,0.3$, and 0.5 . In our experiments trivalent $\mathrm{La}^{3+}$ is chosen to replace the $\mathrm{Sr}^{2+}$ ions because the ionic radius of $\mathrm{La}^{3+}\left(r_{\mathrm{La}^{3+}}=1.36 \AA\right)$ is similar to that of $\mathrm{Sr}^{2+}\left(r_{\mathrm{Sr}^{2+}}=1.44 \AA\right)$. X-ray analysis showed that the lattice parameter slightly decreases from $7.818 \AA(x=0)$ to $7.804 \AA(x=0.5)$ as expected due to the smaller ionic radius of $\mathrm{La}^{3+}$ compared to 


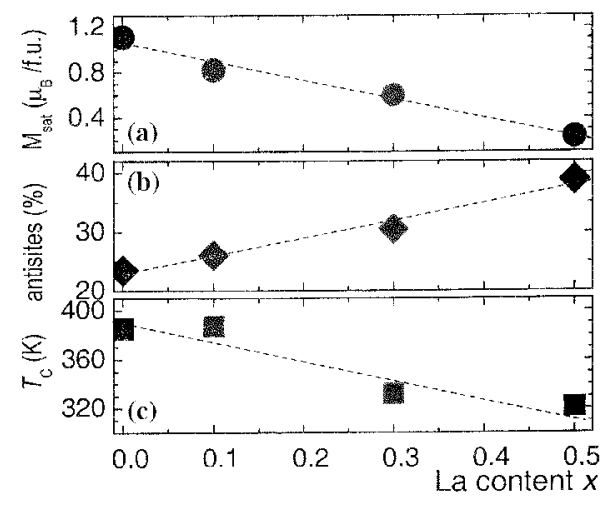

Fig. 9. Variation of the saturation magnetization $M_{\mathrm{sat}}$ at $5 \mathrm{~K}(\mathrm{a})$, the amount of antisite defects (b) and the Curie temperature $T_{C}$ (c) as a function of the La substitution $x$ in $\mathrm{Sr}_{2-x} \mathrm{La}_{x} \mathrm{CrWO}_{6}$. The dotted lines are linear fits to the data.

$\mathrm{Sr}^{2+}$. However, the crystal structure of all samples remained cubic. Figure 9 shows that with increasing La content we observe an increase in antisites, a reduction of the saturation magnetization, and a reduction of the Curie temperature.

At present, we can offer the following plausible explanation. Since La doping is expected to result in a reduction of the differences in the valence states of $\mathrm{Cr}$ and $W$, it evidently results in a reduction of the differences in the ionic radii of $\mathrm{Cr}$ and $\mathrm{W}\left(r_{\mathrm{Cr}^{2}+} / r_{\mathrm{W}^{6+}}=0.73 / 0.63\right.$ and $\left.r_{\mathrm{Cr}^{3+}} / r_{\mathrm{W}^{5+}}=0.615 / 0.62\right)$. Therefore, increasing the doping level results in more similar ionic radii of $\mathrm{Cr}$ and $\mathrm{W}$ paving the way for the creation of $\mathrm{Cr} / \mathrm{W}$ antisites.

We next discuss the observed decrease in $M_{\text {sat }}$ and $T_{\mathrm{C}}$ with increasing La content. Since La substitution causes both electron doping and an increase in antisite defects, an unambiguous explanation of the $M_{\text {sat }}$ and $T_{\mathrm{C}}$ reduction is not possible. With respect to $M_{\text {sat }}$, on the one hand an increasing amount of antisites is expected to reduce $M_{\text {sat }}$ as discussed already above. This is in agreement with other experimental results and recent Monte Carlo simulations [16, 20, 62]. However, on the other hand also within the SFKT model a reduction of the saturation magnetization with increasing electron doping is expected to reduce $M_{\text {sat }}$. According to the illustration given in Fig. 6 it is evident that electron doping in the $\mathrm{Sr}_{2} \mathrm{CrWO}_{6}$ system increases the induced spin-down magnetic moment at the W site, since the electrons are filled into the $W 5 d \downarrow$ band, and thereby reduces $M_{\text {sat }}$ due to the antiferromagnetic sublattice coupling of the $\mathrm{Cr}$ and $\mathrm{W}$ moments.

We finally discuss the influence of La doping on the Curie temperature. In general, both the experimental data on the variation of $T_{\mathrm{C}}$ with electron doping as well as the theoretical interpretation is controversial at present. In single crystals of $\mathrm{Sr}_{2-x} \mathrm{La}_{x} \mathrm{FeMoO}_{6}$ Moritomo et al. have found that $T_{\mathrm{C}}$ does not change as a function of La doping for $x \leq 0.3$ [43]. In contrast, Navarro et al. have reported a 
considerable increase in $T_{\mathrm{C}}$ of about $70 \mathrm{~K}$ in ceramic samples of $\mathrm{Sr}_{2-x} \mathrm{La}_{x} \mathrm{FeMoO}_{6}$ investigating a wider doping range $0 \leq x \leq 1$ [16]. A similar increase has been observed by Serrate et al. for polycrystalline $\left(\mathrm{Sr}_{0.2} \mathrm{Ba}_{0.8}\right)_{2-x} \mathrm{La}_{x} \mathrm{FeMoO}_{6}$ [15]. In our study of the system $\mathrm{Sr}_{2-x} \mathrm{~L}_{x} \mathrm{CrWO}_{6}$ we have found a reduction of $T_{\mathrm{C}}$ of about $80 \mathrm{~K}$ in the doping range $0 \leq x \leq 0.5$.

From the theoretical point of view one expects both an increase and decrease in $T_{\mathrm{C}}$ with increasing doping depending on the model. On the one hand, within the SFKT model [45-47] $T_{\mathrm{C}}$ is expected to be rather reduced than enhanced by electron doping due to the fact that the possible energy gain by shifting electrons from spin-up band into the spin-down band is reduced [44]. This, in turn, reduces the stability of the ferromagnetic phase in agreement with our results. However, the role of the increasing amount of antisites with increasing doping still has to be clarified. On the other hand, in a double exchange type model the increase in the number of conduction electrons promoted by La doping is expected to enhance the double exchange interaction leading to an increase in $T_{\mathrm{C}}$. That is, at first glance the observed decrease in $T_{\mathrm{C}}$ with increasing doping level in the system $\mathrm{Sr}_{2-x} \mathrm{La}_{x} \mathrm{CrWO}_{6}$ seems to support the SFKT model. However, we have to take into account that by La doping besides the number of electrons we also increase the amount of disorder. The latter may weaken the double exchange interaction sufficiently to result in an effective decrease in $T_{\mathrm{C}}$ also within a double exchange based model. Further work is required to clarify this issue in more detail.

\section{Acknowledgment}

This work was supported by the Deutsche Forschungsgemeinschaft and the Bundesministerium fur Bildung und Forschung (project 13N8279). The authors acknowledge fruitful discussions and experimental support by M.S. Brandt, T. Graf, J. Simon, T. Walther, W. Mader, M.S. Ramachandra Rao, D. Topwal, and D.D. Sarma.

\section{References}

[1] F. Galasso, F.C. Douglas, R. Kasper, J. Chem. Phys. 44, 1672 (1966); see also F.S. Galasso, Structure, Properties and Preparation of Perovskite-type Compounds, Pergamon Press, Oxford 1969.

[2] F.K. Patterson, C.W. Moeller, R. Ward, Inorg. Chem. 2, 196 (1963).

[3] J. Longo, R. Ward, J. Am. Chem. Soc. 83, 2816 (1961).

[4] G. Blasse, J. Inorg. Nucl. Chem. 27, 993 (1965).

[5] T. Nakagawa, K. Yoshikawa, S. Nomura, J. Phys. Soc. Jpn. 27, 880 (1969).

[6] K.-I. Kobayashi, T. Kimura, H. Sawada, K. Terakura, Y. Tokura, Nature 395, 677 (1998).

[7] H. Kato, T. Okuda, Y. Okimoto, Y. Tomioka, Y. Takenoya, A. Ohkubo, M. Kawasaki, Y. Tokura, Appl. Phys. Lett. 81, 328 (2002). 
[8] S.A. Wolf, D.D. Awschalom, R.A. Buhrman, J.M. Daughton, S. von Molnar, M.L. Roukes, A.Y. Chtchelkanova, D.M. Treger, Science 294, 1488 (2001).

[9] D.D. Sarma, E.V. Sampathkumaran, Sugata Ray, R. Nagarajan, S. Majumdar, A. Kumar, G. Nalini, T.N. Guru Row, Solid State Commun. 114, 465 (2000).

[10] M. García-Hernández, J.L. Martínez, M.J. Martínez-Lope, M.T. Casais, J.A. Alonso, Phys. Rev. Lett. 86, 2443 (2001).

[11] K.-I. Kobayashi, T. Kimura, Y. Tomioka, H. Sawada, K. Terakura, Y. Tokura, Phys. Rev. B 59, 11159 (1999).

[12] J.B. Philipp, D. Reisinger, M. Schonecke, A. Marx, A. Erb, L. Alff, J. Klein, R. Gross, Appl. Phys. Lett. 79, 3654 (2002).

[13] J.B. Philipp, D. Reisinger, M. Schonecke, A. Marx, A. Erb, L. Alff, R. Gross, J. Appl. Phys. 93, 6853 (2003).

[14] J.B. Philipp, P. Majewski, L. Alff, A. Erb, R. Gross, T. Graf, M.S. Brandt, J. Simon, T. Walther, W. Mader, D. Topwal, D.D. Sarma, Phys. Rev. B, accepted for publication, 2003.

[15] D. Serrate, J.M. De Teresa, J. Blasco, M.R. Ibarra, L. Morellón, C. Ritter, Appl. Phys. Lett. 80, 4573 (2002).

[16] J. Navarro, C. Frontera, L. Balcells, B. Martínez, J. Fontcuberta, Phys. Rev. B 64, 092411 (2001).

[17] J.H. Park, S.K. Kwon, B.I. Min, Phys. Rev. B 65, 174401 (2002).

[18] K. Ueda, H. Tabata, T. Kawai, Science 280, 1064 (1998).

[19] W.E. Pickett, Phys. Rev. B 57, 10613 (1998); see also Science 281, 1571a (1998).

[20] L. Balcells, J. Navarro, M. Bibes, A. Roig, B. Martínez, J. Fontcuberta, Appl. Phys. Lett. 78, 781 (2001).

[21] J. Gopalakrishnan, A. Chattopadhyay, S.B. Ogale, T. Venkatesan, R.L. Greene, A.J. Millis, K. Ramesha, B. Hannoyer, G. Marest, Phys. Rev. B 62, 9538 (2000).

[22] W. Westerburg, O. Lang, C. Ritter, C. Felser, W. Tremel, G. Jakob, Solid State Commun. 122, 201 (2002).

[23] W. Prellier, V. Smolyaninova, A. Biswas, C. Galley, R.L. Greene, K. Ramesha, J. Gopalakrishnan, J. Phys., Condens. Matter 12, 965 (2000).

[24] P.D. Battle, T.C. Gibb, A.J. Herod, S.-H. Kim, P.M. Munns, J. Mater. Chem. 5, 865 (1995).

[25] R. Rodriguez, A. Fernandez, A. Isalgue, J. Rodriguez, A. Labarta, J. Tejada, X. Obradors, J. Phys. 18, L401 (1985).

[26] T.C. Gibb, J. Mater. Chem. 3, 441 (1993).

[27] A.K. Azad, S.-G. Erikson, A. Mellergard, S.A. Ivanov, J. Eriksen, H. Rundlöf, Mater. Res. Bull. 37, 1797 (2002).

[28] H. Kawanaka, I. Hase, S. Toyama, Y. Nishihara, Physica B 284-288, 1428 (2000); see also Physica B 281-282, 518 (2000).

[29] K.-I. Kobayashi, T. Okuda, Y. Tomioka, T. Kimura, Y. Tokura, J. Magn. Magn. Mater. 218, 17 (2000).

[30] M.J. Martinez-Lope, J.A. Alonso, M.T. Casais, M.T. Fernandez-Diaz, Z. Naturforsch. B 58, 127 (2003). 
[31] A.K. Azad, S.A. Ivanov, S.-G. Eriksson, J. Eriksen, H. Rundlöf, R. Mathieu, P. Svedlindh, Mater. Res. Bull. 36, 2215 (2000).

[32] A.K. Azad, S.A. Ivanov, S.-G. Eriksson, J. Eriksen, H. Rundlöf, R. Mathieu, P. Svedlindh, Mater. Res. Bull. 36, 2485 (2000).

[33] A.K. Azad, S. Ivanov, S.-G. Eriksson, H. Rundlöf, J. Eriksen, R. Mathieu, P. Svedlindh, J. Magn. Magn. Mater. 237, 124 (2001).

[34] C.R. Wiebe, J.E. Greedan, G.M. Luke, Phys. Rev. B 65, 144413 (2002).

[35] D. Iwanga, Y. Inaguma, M. Itoh, Mater. Res. Bull. 35, 449 (2000).

[36] H. Karundasa, Q. Huang, B.G. Ueland, P. Schiffer, R.J. Cava, Proc. Natl. Acad. Sci. 100, 8097 (2003).

[37] A.P. Ramirez, Ann. Rev. Mater. Sci. 24, 453 (1994).

[38] J.E. Greendan, J. Mater. Chem. 11, 37 (2001).

[39] R. Moessner, Can. J. Phys. 79, 37 (2001); see also Phys. Rev. Lett. 93, 3293 (1999).

[40] M.J. Harris, S.T. Bramwell, P.C.W. Holdsworth, J.D.M. Champion, Phys. Rev. Lett. 79, 2554 (1997); see also Phys. Rev. Lett. 81, 4496 (1998) and Nature 399, 311 (1999).

[41] A.P. Ramirez, A. Hayashi, R.J. Cava, R. Siddharthan, B.S. Shastry, Nature 399, 333 (1999); see also Phys. Rev. Lett. 89, 067202 (2002).

[42] Y. Moritomo, Sh. Xu, A. Machida, T. Akimoto, E. Nishibori, M. Takata, M. Sakata, Phys. Rev. B 61, R7827 (2000).

[43] Y. Moritomo, Sh. Xu, T. Akimoto, A. Machida, N. Hamada, K. Ohoyama, E. Nishibori, M. Takata, M. Sakata, Phys. Rev. B 62, 14224 (2000).

[44] K. Phillips, A. Chattopadhyay, A.J. Millis, Phys. Rev. B 67, 125119 (2003).

[45] D.D. Sarma, P. Mahadevan, T. Saha-Dasgupta, Sugata Ray, A. Kumar, Phys. Rev. Lett. 85, 2549 (2000); see also Curr. Opinion in Solid State Mater. Sci. 5, 261 (2001).

[46] J. Kanamori, K. Terakura, J. Phys. Soc. Jpn. 70, 1433 (2001).

[47] Z. Fang, K. Terakura, J. Kanamori, Phys. Rev. B 63, R180407 (2001).

[48] C. Zener, Phys. Rev. 82, 403 (1951).

[49] P.W. Anderson, H. Hasegawa, Phys. Rev. 100, 67 (1955).

[50] J.H. Jung, S.-J. Oh, M.W. Kim, T.W. Noh, J.-Y. Kim, J.-H. Park, H.-J. Lin, C.T. Chen, Y. Moritomo, Phys. Rev. B 66, 104415 (2002).

[51] H. Wu, Phys. Rev. B 64, 125126 (2001).

[52] M. Tovar, M.T. Causa, A. Butera, J. Navarro, B. Martinez, J. Fontcuberta, M.C.G. Passeggi, Phys. Rev. B 66, 024409 (2002).

[53] Horng-Tay Jeng, G.Y. Guo, Phys. Rev. B 67, 094438 (2003).

[54] R.P. Borges, R.M. Thomas, C. Cullinan, J.M.D. Coey, R. Suryanarayanan, L. Ben-Dor, L. Pinsard-Gaudart, A. Revcolevschi, J. Phys., Condens. Matter 11, L445 (1999).

[55] C. Ritter, M.R. Ibarra, L. Morellón, J. Blasco, J. Garcia, J.M. De Teresa, J. Phys., Condens. Matter 12, 8295 (2000). 
[56] W.H. Song, J.M. Dai, S.L. Ye, K.Y. Wang, J.J. Du, Y.P. Sun, J. Appl. Phys. 89, 7678 (2001).

[57] Bog-Gi Kim, Yew-San Hor, S.-W. Cheong, Appl. Phys. Lett. 79, 388 (2001).

[58] H.Y. Hwang, S.-W. Cheong, P.G. Radaelli, M. Marezio, B. Batlogg, Phys. Rev. Lett. 75, 914 (1995).

[59] J.P. Zhou, J.T. McDevitt, J.S. Zhou, H.Q. Yin, J.B. Goodenough, Y. Gim, Q.X. Jia, Appl. Phys. Lett.75, 1146 (1999).

[60] H. Kato, T. Okuda, Y. Okimoto, Y. Tomioka, K. Oikawa, T. Kamiyama, Y. Tokura, Phys. Rev. B 65, 144404 (2002).

[61] M. Imada, A. Fujimori, Y. Tokura, Rev. Mod. Phys. 30, 1039 (1998).

[62] T. Saha-Dasgupta, D.D. Sarma, Phys. Rev. B 64, 064408 (2001). 